\title{
Jamming Games for Power Controlled Medium Access with Dynamic Traffic
}

\author{
Yalin Evren Sagduyu \\ Intelligent Automation Inc. \\ Rockville, MD 20855, USA, and \\ Institute for Systems Research \\ University of Maryland \\ College Park, MD 20742, USA \\ Email: yalinsagduyu@ieee.org
}

\author{
Randall A. Berry \\ Department of Electrical Engineering \\ and Computer Science \\ Northwestern University \\ Evanston, IL 60208, USA \\ Email: rberry@ece.northwestern.edu
}

\author{
Anthony Ephremides \\ Department of Electrical \\ and Computer Engineering, and \\ Institute for Systems Research \\ University of Maryland \\ College Park, MD 20742, USA \\ Email: etony@umd.edu
}

\begin{abstract}
Due to the broadcast nature of the wireless medium, wireless networks are highly susceptible to jamming attacks. Such attacks are often studied in a game theoretic framework under the assumption of uninterrupted traffic subject to continuous jamming opportunities. Instead, we analyze the effect of dynamically changing traffic on jamming games for power controlled medium access. Random packet arrivals raise the possibility that the transmitter queues may be empty when jamming attacks start and thus waste the energy of jammers. We consider a noncooperative game in which transmitters and jammers select their transmission power to balance the transmission cost subject to delay and energy constraints. We show that jammers incur a significant performance loss when they do not have knowledge of transmitter queue states. Dynamic traffic increases the immunity to jamming attacks and gives insights into defense mechanisms.

Index Terms-Jamming; Non-cooperative game; Power control; Medium access control; Queue stability; Energy; Delay.
\end{abstract}

\section{INTRODUCTION}

Wireless networks are inherently based on broadcast transmissions thereby increasing their vulnerability to denial of service attacks via jamming at the medium access control (MAC) layer. Such jamming problems can be modeled as a non-cooperative game [1]-[10] in which "selfish" transmitters try to maximize their own performance, while "malicious" jamming nodes attempt to degrade the system performance.

It is typically assumed that the (selfish) transmitters always have packets available to transmit. However, in most networks, packet traffic will vary dynamically, i.e., transmitters may receive bursty traffic from applications or may receive random traffic to be relayed. With dynamic traffic, a jammer will only be successful when the transmitter queues are not empty. However, in a distributed wireless network, jammers may not have access to the queue state of transmitters, and transmitters may benefit from concealing this information from jammers. In this paper we consider game theoretic models for jamming in wireless networks with dynamic traffic and asses the effect of random traffic on the effectiveness of jamming attacks.

R. A. Berry was supported in part by NSF under grant CCF-0905224.

A. Ephremides was supported in part by NSF under grants CCF-0905204 and CCF-0728966, by ONR under grant N00140911164, and by U.S. Army under MURI grant W911NF-05-1-0246.
We assume that transmitters are sending randomly arriving traffic to receivers and jammers attempt to interfere with these transmissions in form of a denial of service attack. We assume a power controlled MAC where nodes select their individual transmission powers. The interactions between transmitters and jammers are modeled as a non-cooperative game. In these games, each transmitter seeks to minimize its average energy cost subject to a target rate constraint, which can be modeled as an upper bound on the average packet delay. On the other hand, each jammer seeks to maximize the average energy costs of transmitters subject to its own average energy constraint.

We start by focusing on a single transmitter-jammer pair at one receiver and subsequently generalize the model to arbitrary sets of transmitters and jammers communicating to a single receiver and then to multiple channels and receivers. In the latter cases, we allow a transmitter-jammer pair to choose at which channels and receivers they transmit and interfere. In each case, we compare the performance when jammers know the queue state of transmitters and do not.

This performance is measured by evaluating the Nash equilibrium strategies of the resulting games and calculating the maximum feasible throughput of the selfish nodes in stable operation. We show that jammers experience performance loss (i.e., the feasible throughput is larger and the average energy cost is smaller for transmitters) without knowledge of whether the transmitter queues are empty or not, i.e., traffic uncertainty is beneficial to transmitters in power control games (Similar results apply to other MAC models, e.g., random access games where nodes select their transmission probabilities [8].). This phenomenon is similar to the performance loss of jammers under type uncertainty [9], i.e., when nodes do not know whether the opponents are selfish or malicious.

In terms of related work, an information-theoretic framework has been considered in [1], where a transmitter-jammer pair balances the mutual information as the objective function. The model has been extended as a non-cooperative game to MIMO fading channels [2] and to MAC channels with two users in the presence of a jammer [3]. The energy cost has been incorporated into jamming games over multiple Gaussian channels in [4], where the jammer signal is treated as noise, 
and the effects of random channels on jamming games have been analyzed in [5]. In addition, power control games have been considered in [6], [7] where transmitters and jammers choose their transmission powers to balance their throughput rewards and energy costs. All this previous work was based on the common assumption of backlogged transmitters.

The rest of this paper is organized as follows. Section II introduces the system model for one transmitter-jammer pair at a single receiver. The case without queue state information at the jammer is studied in Section III and it is followed in Section IV by the case where the jammer has access to the transmitter's queue state information. Section V imposes an average packet delay constraint on the equilibrium strategies derived in Sections III-IV, and the feasible rate performance is compared. Then, we extend the analysis to an arbitrary number of transmitters and jammers in Section VI, and we consider the case with multiple channels at different receivers in Section VII. We conclude the paper in Section VIII.

\section{Single Transmitter-Jammer Pair Model}

We start by examining a scenario with one transmitter (node 1) and one jammer (node 2). Packets randomly arrive at node 1 and are buffered in a queue until they can be transmitted to a single receiver over a single channel. Node 2 does not have its own traffic and simply wants to jam the transmissions of node 1. We make two simplifying assumptions: (A1) we assume a synchronous slotted system, in which each packet transmission (or jamming attempt) takes one time slot, and (A2) we assume that the packet arrivals are independent and identically distributed over time with rate $\lambda$ (packets per slot). ${ }^{1}$

We consider a non-cooperative game in which the two nodes are players. Each player $i$ spreads their signal over a common frequency band with power $P_{i}$ and treats interference as noise. The objectives of node 1 are: $(i)$ to meet a target minimum rate constraint $r(\lambda, D)$ which could depend on a Quality of Service parameter $D,{ }^{2}$ and (ii) minimize the average energy cost subject to constraint $(i)$. The objectives of node 2 are: $(i)$ to maximize the average transmission cost of node 1 , and (ii) satisfy an upper bound $E_{2}$ on its own average energy cost.

Node 1 transmits with power $P_{1}$ only if it has a packet in its queue. Node 2 transmits at a constant power $P_{2}$ creating interference that is treated as noise by node 1 . We model the resulting service rate using the Shannon rate:

$$
\mu\left(P_{1}, P_{2}\right)=\frac{1}{2} \log _{2}\left(1+\frac{h_{1} P_{1}}{h_{2} P_{2}+N}\right),
$$

where $h_{i}$ is the channel gain for user $i$ and $N$ is the noise

\footnotetext{
${ }^{1}$ Both of these limit the ability of the jammer; for example. (A1) precludes the jammer from waiting to detect the start of a transmission before jamming, while (A2) makes it more difficult for the jammer to infer the queue state of the transmitter than, for example, a case where packets arrive in batches.

${ }^{2}$ In Section V, we look at a specific model where $D$ corresponds to a packet delay constraint, however the following results hold for any such function for which $r(\lambda, D)>\lambda$, which ensures that when this constraint is satisfied, node 1 's queue is stable.
}

power. $^{3}$ The transmission energy cost of node $i=1,2$ is the power $P_{i}$, whenever node $i$ transmits. All parameters $h_{i}, E_{i}$, $i=1,2$, and $N$ are fixed and known to each node.

\section{No Queue StATE Information AT JAMMER}

We assume that jammer node 2 does not know whether transmitter node 1 has a packet to transmit, or not. We consider a one-shot game in which the jammer chooses a fixed strategy $P_{2}$ for all time. Packets of node 1 are served with rate $\mu\left(P_{1}, P_{2}\right)$ given by $(1)$. The probability that there exists a packet in node 1 's queue is given by the utilization factor $\frac{\lambda}{\mu\left(P_{1}, P_{2}\right)}$ according to Little's result [12]. Node 1's average transmission cost is $\frac{\lambda}{\mu\left(P_{1}, P_{2}\right)} P_{1}$. Hence, node 1 's objective in the game is to solve the following optimization problem

$$
\begin{aligned}
\max _{P_{1} \geq 0} & u_{1}\left(P_{1}, P_{2}\right):=-\frac{\lambda}{\mu\left(P_{1}, P_{2}\right)} P_{1} \\
\text { subject to } & \mu\left(P_{1}, P_{2}\right) \geq r(\lambda, D) .
\end{aligned}
$$

Node 2 chooses power $P_{2} \geq 0$ to maximize the average energy cost of node 1 subject to its own energy constraint $P_{2} \leq E_{2}$, namely solves the following optimization problem

$$
\begin{aligned}
\max _{P_{2} \geq 0} & u_{2}\left(P_{1}, P_{2}\right):=\frac{\lambda}{\mu\left(P_{1}, P_{2}\right)} P_{1} \\
\text { subject to } & P_{2} \leq E_{2} .
\end{aligned}
$$

We characterize the outcome of this game by finding the Nash equilibrium strategies $P_{i}^{*}$ of node $i=1,2$, which satisfy

$$
u_{i}\left(P_{i}^{*}, P_{-i}^{*}\right) \geq u_{i}\left(P_{i}, P_{-i}^{*}\right),\left(P_{i}^{*}, P_{-i}^{*}\right) \in \mathcal{C}, i=1,2,
$$

for any strategy $P_{i}, i=1,2$, where $P_{-i}$ is the strategy of the node other than node $i$ and $\mathcal{C}$ is the constraint set given by

$$
\mathcal{C}=\left\{P_{i} \geq 0, i=1,2, \mu\left(P_{1}, P_{2}\right) \geq r(\lambda, D), P_{2} \leq E_{2}\right\} .
$$

At Nash equilibrium, node 2 continuously transmits with the largest possible power $P_{2}=E_{2}$ that satisfies the average energy constraint. Given a choice of $P_{2}$, node 1's utility is strictly decreasing in $P_{1}$ and so node 1 will select its power $P_{1}$ to satisfy $\mu_{1}\left(P_{1}, P_{2}\right)=r(\lambda, D)$. Hence, in this case there is always a unique Nash equilibrium given by

$$
P_{1}^{*}=\frac{1}{h_{1}}\left(2^{2 r(\lambda, D)}-1\right)\left(h_{2} E_{2}+N\right), \quad P_{2}^{*}=E_{2} .
$$

The model assumes that the jammer transmits with a fixed power $P_{2}$. If the jammer chooses $P_{2}$ randomly, the utility functions need to be formulated as expected payoffs over $P_{2}$. In that case, the jammer seeks to maximize its expected utility $E\left[u_{2}\left(P_{1}, P_{2}\right)\right]$ or to minimize the expected service rate $E\left[\mu\left(P_{1}, P_{2}\right)\right]$ of the transmitter, where the expectation is taken over $P_{2}$. Since $\mu\left(P_{1}, P_{2}\right)$ is convex in $P_{2}, E\left[\mu\left(P_{1}, P_{2}\right)\right] \geq$ $\mu\left(P_{1}, E\left[P_{2}\right]\right)$ follows from Jensen's inequality. Therefore, the

\footnotetext{
${ }^{3}$ This rate can be approached only under sufficiently long blocks of codes. However, the performance gap of practical codes from the informationtheoretic limits is small for fading channels with sufficiently long coherence times [11]. We use (1) as the maximum reference rate. The results would extend to other (suboptimal) rate formulations depending on the Signal-toInterference-plus-Noise-Ratio given by $\frac{h_{1} P_{1}}{h_{2} P_{2}+N}$.
} 
average service rate cannot be reduced by randomizing $P_{2}$ and the best strategy for the jammer is to choose a fixed transmission power under the average energy constraint.

Next, we introduce an average energy constraint $E_{1}$ on node 1 given by $\frac{\lambda}{r(\lambda, D)} P_{1} \leq E_{1}$ (since it transmits with power $P_{1}$ with probability $\frac{\lambda}{r(\lambda, D)}$ only when its queue is not empty). Then, from (6) the set of node 1's feasible rates $\mathcal{S}$ is

$$
\mathcal{S}=\left\{\lambda \geq 0: \quad \lambda \leq \frac{h_{1} E_{1} r(\lambda, D)}{\left(2^{2 r(\lambda, D)}-1\right)\left(h_{2} E_{2}+N\right)}\right\} .
$$

If the arrival rate is not feasible, then the transmitter cannot satisfy the target rate and delay constraints, and the Nash equilibrium does not exist for the jamming game.

\section{Queue State Information at JAMmer}

Now we turn to the case, where the jammer node 2 knows whether transmitter node 1 has a packet to transmit, or not. This also includes the special case when the transmitter queue is backlogged. In this case, node 2 can adjust its strategy so as to conserve energy (by not transmitting) when node 1 does not have a packet. Node 2 transmits with power $P_{2} \geq 0$ only if the queue of node 1 is not empty, which occurs with probability $\frac{\lambda}{\mu\left(P_{1}, P_{2}\right)}$; otherwise, node 2 stays idle. Therefore, node 2 now seeks to solve the following optimization problem

$$
\begin{aligned}
\max _{P_{2} \geq 0} & u_{2}\left(P_{1}, P_{2}\right):=\frac{\lambda}{\mu\left(P_{1}, P_{2}\right)} P_{1} \\
\text { subject to } & \frac{\lambda}{\mu\left(P_{1}, P_{2}\right)} P_{2} \leq E_{2} .
\end{aligned}
$$

This is accomplished by choosing the largest possible $P_{2}$. Node 1 faces the same objective as in (2) and thus again will choose $P_{1}$ to satisfy $\mu\left(P_{1}, P_{2}\right)=r(\lambda, D)$. Hence, the unique Nash equilibrium for this game is given by

$$
\begin{aligned}
& P_{1}^{*}=\frac{1}{h_{1}}\left(2^{2 r(\lambda, D)}-1\right)\left(h_{2} E_{2} \frac{r(\lambda, D)}{\lambda}+N\right), \\
& P_{2}^{*}=E_{2} \frac{r(\lambda, D)}{\lambda} .
\end{aligned}
$$

Given the average energy constraint $\frac{\lambda}{r(\lambda, D)} P_{1} \leq E_{1}$, the set of feasible arrival rates for node 1 is given by

$$
\mathcal{S}=\left\{\lambda \geq 0: \lambda \leq \frac{h_{1} E_{1} r(\lambda, D)}{\left(2^{2 r(\lambda, D)}-1\right)\left(h_{2} E_{2} \frac{r(\lambda, D)}{\lambda}+N\right)}\right\} .
$$

The maximum feasible rate in (11) is smaller than the maximum feasible rate in (7), showing that the jamming attack is more effective given the queue state information. The stable rates in (7) and (11) approach each other, as $\lambda$ increases to $r(\lambda, D)$. In this limit, the transmitter queue becomes saturated and so queue state information is of little value.

\section{Jamming Games under a Delay Constraint}

In this section, we examine a simple model for relating the target rate constraint $r(\lambda, D)$ in the previous games to an average delay constraint $D$. This provides an example to show how the resulting equilibria may vary depending on such a delay constraint. The service time of node 1's packets is deterministic and given by $\mu\left(P_{1}, P_{2}\right)$. Then, with Poisson arrivals node 1's packets can be viewed as waiting in a $M / D / 1$ queue. Using the Pollaczek-Khinchin formula [12], the average total packet delay $T\left(\lambda, P_{1}, P_{2}\right)$ is given by

$$
T\left(\lambda, P_{1}, P_{2}\right)=\frac{2 \mu\left(P_{1}, P_{2}\right)-\lambda}{2 \mu\left(P_{1}, P_{2}\right)\left(\mu\left(P_{1}, P_{2}\right)-\lambda\right)} .
$$

Hence, the delay constraint $T\left(\lambda, P_{1}, P_{2}\right) \leq D$ corresponds to the service rate constraint $\mu\left(P_{1}, P_{2}\right) \geq r(\lambda, D)$, where

$$
r(\lambda, D)=\frac{\lambda D+1+\sqrt{\lambda^{2} D^{2}+1}}{2 D} .
$$

The maximum feasible throughput rates (7) and (11) are compared in Figure 1. The transmitter can significantly improve its maximum feasible throughput if the jammer does not know the transmitter queue state and when the delay constraint is small. However, this rate improvement decreases and the maximum feasible rate for the two cases approach each other, as $D$ increases and the transmitter queue becomes backlogged. A similar behavior is also observed as $h_{2}$ decreases such that the jamming effect disappears.

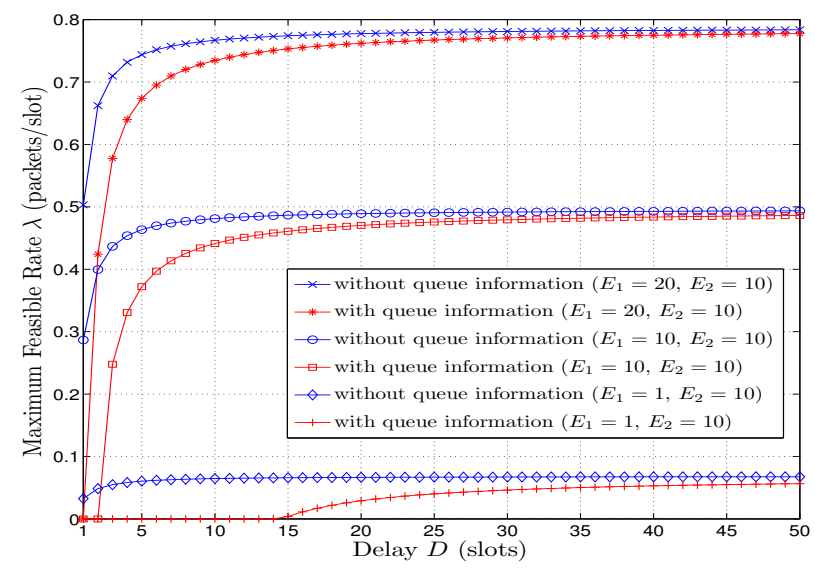

Fig. 1. The maximum feasible throughput for $h_{1}=1, h_{2}=1$, and $N=0.1$.

\section{Extension to AN Arbitrary Number of Nodes}

Next we consider $n_{T}$ nodes transmitting to a single receiver and $n_{J}$ jammer nodes with the objectives of interfering with the packet transmissions. We assume a symmetric system, in which the transmitters and jammers are subject to common average energy costs $E_{T}$ and $E_{J}$, respectively. The total arrival rate is $\lambda$ and it is equally shared by $n_{T}$ transmitters. Each transmitter is subject to the same target rate constraint of $r\left(\frac{\lambda}{n_{T}}, D\right)$, which again could depend on a delay parameter $D$. We focus on characterizing symmetric equilibria, under which all transmitters or jammers chose the same strategy.

All transmitters and jammers transmit with powers $P_{T}$ and $P_{J}$ over channels with gains $h_{T}$ and $h_{J}$, respectively. The service rate of any transmitter queue is given by

$$
\mu\left(P_{T}, P_{J}\right)=\frac{1}{2} \log _{2}\left(1+\frac{h_{T} P_{T}}{\tilde{P}\left(P_{T}, P_{J}\right)}\right),
$$


where the total interference and noise power, $\tilde{P}$, is

$$
\tilde{P}\left(P_{T}, P_{J}\right)=h_{T}\left(n_{T}-1\right) P_{T}+h_{J} n_{J} P_{J}+N .
$$

The symmetric equilibrium strategy for any transmitter is

$$
P_{T}^{*}=\frac{1}{h_{T}}\left(2^{2 r\left(\frac{\lambda}{n_{T}}, D\right)}-1\right) \tilde{P}\left(P_{T}^{*}, P_{J}^{*}\right),
$$

and the symmetric equilibrium strategy for any jammer is

$$
P_{J}^{*}=E_{J}
$$

if jammers do not have the queue state information of the transmitters. Otherwise, jammers transmit with higher power

$$
P_{J}^{*}=\frac{E_{J} n_{T} r\left(\frac{\lambda}{n_{T}}, D\right)}{\lambda} .
$$

Hence, as with the single transmitter-jammer pair model, the transmission power of jammers is increased compared to the case without queue state information. This reduces the maximum feasible rates of the transmitters (each with average energy constraint $E_{T}$ ), as shown in Figure 2 for $n_{T}=1$.

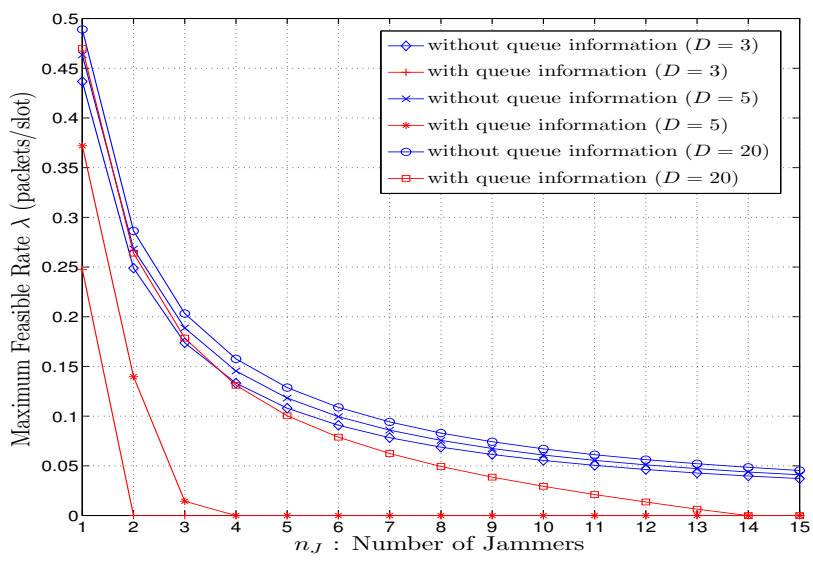

Fig. 2. The maximum feasible throughput for $E_{T}=10, E_{J}=10, h_{T}=1$, $h_{J}=1$, and $N=0.1$.

\section{JAMming AT Multiple ReCEIVERS}

We now extend the model in Section II (for a single transmitter-jammer pair) to one with $n$ receivers with independent channels from transmitter node 1 and jammer node 2. Node 1 allocates its transmission resources among these $n$ channels and node 2 attempts to jam the transmissions on each channel. Node 2 is limited by an average cost over all channels. This may also model transmissions to a single receiver over multiple sub-carriers as in orthogonal frequency division multiplexing (OFDM) systems. Node 1 buffers the incoming packets to be transmitted on each channel $i \in\{1, \ldots, n\}$ in a separate queue with arrival rate $\lambda_{i}$ and service rate $\mu_{i}$. The packet traffic is split to the individual channels (e.g., with different priorities or Quality of Service requirements) such that $\sum_{i=1}^{n} \lambda_{i}=\lambda$. We assume that the packets transmitted on channel $i$ are subject to the target service rate $r_{i}\left(\lambda_{i}, D_{i}\right)$.

\section{A. Fixed Traffic Rates on Channels}

First, we assume that the traffic rates $\left\{\lambda_{i}\right\}_{i=1}^{n}$ and delay constraints $\left\{D_{i}\right\}_{i=1}^{n}$ are predetermined with the target service rates $\left\{r_{i}\left(\lambda_{i}, D_{i}\right)\right\}_{i=1}^{n}$ so that we have $\lambda_{i}<r_{i}\left(\lambda_{i}, D_{i}\right)$ for each channel $i=1, \ldots, n$. The service rate on channel $i$ is given by

$$
\mu_{i}\left(P_{1, i}, P_{2, i}\right)=\frac{1}{2} \log _{2}\left(1+\frac{h_{1, i} P_{1, i}}{h_{2, i} P_{2, i}+N_{i}}\right),
$$

where $P_{j, i}$ and $h_{j, i}$ are the transmission power and the gain of node $j$ on channel $i$, respectively, and $N_{i}$ is the noise power on channel $i$. All these system parameters are fixed and known to both nodes. Node 1 chooses the transmission powers $P_{1, i} \geq 0$, $i=1, \ldots, n$, to maximize the utility

$$
\begin{aligned}
u_{1}\left(\left\{P_{1, i}, P_{2, i}\right\}_{i=1}^{n}\right)= & -\sum_{i=1}^{n} \frac{\lambda_{i}}{\mu_{i}\left(P_{1, i}, P_{2, i}\right)} P_{1, i} \\
\text { subject to } \quad & \mu_{i}\left(P_{1, i}, P_{2, i}\right) \geq r_{i}\left(\lambda_{i}, D_{i}\right) .(
\end{aligned}
$$

Given the equilibrium power $P_{2, i}^{*}$ of node 2 , node 1 chooses in Nash equilibrium the smallest possible power $P_{1, i}^{*}=$ $\left(2^{2 r_{i}\left(\lambda_{i}, D_{i}\right)}-1\right)\left(\frac{h_{2, i} P_{2, i}^{*}+N_{i}}{h_{1, i}}\right)$ to satisfy the rate constraint on each channel $i$ with equality, i.e., $\mu\left(P_{1, i}^{*}, P_{2, i}^{*}\right)=r_{i}\left(\lambda_{i}, D_{i}\right)$.

If node 2 does not know whether node 1 has a packet to transmit on each channel or not, it maximizes the utility

$$
\begin{aligned}
u_{2}\left(\left\{P_{1, i}, P_{2, i}\right\}_{i=1}^{n}\right)= & \sum_{i=1}^{n} \frac{\lambda_{i}}{\mu_{i}\left(P_{1, i}, P_{2, i}\right)} P_{1, i} \\
\text { subject to } & \sum_{i=1}^{n} P_{2, i} \leq E_{2} .
\end{aligned}
$$

If equilibrium strategies $P_{1, i}^{*}$ and $P_{2, i}^{*}$ satisfy $\mu\left(P_{1, i}^{*}, P_{2, i}^{*}\right)=$ $r_{i}\left(\lambda_{i}, D_{i}\right)$, average energy is $\sum_{i=1}^{n} f_{1}\left(\lambda_{i}, D_{i}\right)\left(P_{2, i}^{*}+\frac{N_{i}}{h_{2, i}}\right)$ for node 1 , where $f_{1}\left(\lambda_{i}, D_{i}\right)=\frac{\left(2^{2 r_{i}\left(\lambda_{i}, D_{i}\right)}-1\right) h_{2, i}}{h_{i, 1}}\left(\frac{\lambda_{i}}{r_{i}\left(\lambda_{i}, D_{i}\right)}\right)$. From (20), node 2 assigns all available power to one particular channel $i^{*}$ to maximize node 1's average energy:

$$
P_{2, i^{*}}^{*}=E_{2} \quad \text { for } \quad i^{*}=\underset{i=1, \ldots, n}{\arg \max } f_{1}\left(\lambda_{i}, D_{i}\right),
$$

If node 2 knows whenever node 1 has a packet to transmit on each channel, then the utility of node 2 is changed to

$$
\begin{aligned}
u_{2}\left(\left\{P_{1, i}, P_{2, i}\right\}_{i=1}^{n}\right)= & \sum_{i=1}^{n} \frac{\lambda_{i}}{\mu_{i}\left(P_{1, i}, P_{2, i}\right)} P_{1, i} \\
\text { subject to } & \sum_{i=1}^{n} \frac{\lambda_{i}}{\mu_{i}\left(P_{1, i}, P_{2, i}\right)} P_{2, i} \leq E_{2} .
\end{aligned}
$$

The jammer assigns all power to a particular channel $i^{*}$ :

$P_{2, i^{*}}^{*}=E_{2} \frac{r_{i^{*}}\left(\lambda_{i^{*}}, D_{i^{*}}\right)}{\lambda_{i^{*}}}$ for $i^{*}=\underset{i=1, \ldots, n}{\arg \max } f_{2}\left(\lambda_{i}, D_{i}\right)$,

where $f_{2}\left(\lambda_{i}, D_{i}\right)=\frac{\left(2^{2 r_{i}\left(\lambda_{i}, D_{i}\right)}-1\right) h_{2, i}}{h_{i, 1}}$ such that node 1's average energy is written as $\sum_{i=1}^{n} f_{2}\left(\lambda_{i}, D_{i}\right)\left(P_{2, i}^{*}+\frac{N_{i}}{h_{2, i}}\right)$,

In both cases with and without queue state information at the jammer, if $i^{*}$ is not unique in (22) and (24), the jammer divides its power arbitrarily among channels $i^{*}$. For $P_{2, i}^{*}$ given by (22) or (24), node 1 chooses $P_{1, i}^{*}$ to satisfy the target rate constraint on each channel $i$ with equality. 


\section{B. Optimal Traffic Rates on Channels}

Next, we consider the case in which node 1 further optimizes the channel rates $\left\{\lambda_{i}\right\}_{i=1}^{n}$. Node 1 chooses the transmission powers $\left\{P_{1, i}\right\}_{i=1}^{n}$ and channel rates $\left\{\lambda_{i}\right\}_{i=1}^{n}$, where $P_{1, i} \geq 0, \lambda_{i} \geq 0$ and $\sum_{i=1}^{n} \lambda_{i}=\lambda$, to maximize the utility $u_{1}\left(\left\{P_{1, i}, \lambda_{i}, P_{2, i}\right\}_{i=1}^{n}\right)=-\sum_{i=1}^{n} \frac{\lambda_{i}}{\mu_{i}\left(P_{1, i}, P_{2, i}\right)} P_{1, i}$, where $P_{1, i}=\left(2^{2 r_{i}\left(\lambda_{i}, D_{i}\right)}-1\right)\left(\frac{h_{2, i} P_{2, i}+N_{i}}{h_{1, i}}\right)$, subject to $\mu_{i}\left(P_{1, i}, P_{2, i}\right) \geq r_{i}\left(\lambda_{i}, D_{i}\right)$ and $\lambda_{i}<\mu_{i}\left(P_{1, i}, P_{2, i}\right)$.

If node 2 does not have the queue state information, it maximizes the utility $u_{2}\left(\left\{P_{1, i}, \lambda_{i}, P_{2, i}\right\}_{i=1}^{n}\right)=\sum_{i=1}^{n} \frac{\lambda_{i}}{r_{i}\left(\lambda_{i}, D_{i}\right)} P_{1, i}$ subject to $\sum_{i=1}^{n} P_{2, i} \leq E_{2}$. In Nash equilibrium, node 1 chooses $\left\{\lambda_{i}\right\}_{i=1}^{n}$ to make node 2 indifferent to its strategies $\left\{P_{2, i}\right\}_{i=1}^{n}$. This is realized by choosing the multiplying terms $\left\{f_{1}\left(\lambda_{i}, D_{i}\right)\right\}_{i=1}^{n}$ of $\left\{P_{2, i}\right\}_{i=1}^{n}$ in utility $u_{2}\left(\left\{P_{1, i}, \lambda_{i}, P_{2, i}\right\}_{i=1}^{n}\right)$ equal to each other subject to $\sum_{i=1}^{n} \lambda_{i}=\lambda$. At Nash equilibrium node 2 chooses $\left\{P_{2, i}^{*}\right\}_{i=1}^{n}$ such that $\sum_{i=1}^{n} P_{2, i}^{*}=E_{2}$ and the equilibrium strategies $\left\{\lambda_{i}^{*}\right\}_{i=1}^{n}$ of node 1 minimize its average energy cost $\sum_{i=1}^{n} f_{1}\left(\lambda_{i}, D_{i}\right)\left(P_{2, i}^{*}+\frac{N_{i}}{h_{2, i}}\right)$.

If the jammer has the queue state information, the energy constraint is changed to $\sum_{i=1}^{n} \frac{\lambda_{i}}{\mu_{i}\left(P_{1, i}, P_{2, i}\right)} P_{2, i} \leq E_{2}$ and $f_{1}\left(\lambda_{i}, D_{i}\right)$ is replaced by $f_{2}\left(\lambda_{i}, D_{i}\right)$, as given in Section VII$A$. In both cases, node 1 chooses $P_{1, i}$ to satisfy $\mu_{i}\left(P_{1, i}, P_{2, i}^{*}\right)=$ $r_{i}\left(\lambda_{i}^{*}, D_{i}\right)$ for the equilibrium strategies $P_{2, i}^{*}$ and $\lambda_{i}^{*}$.

For illustration purposes, we consider two receivers. The Nash equilibrium solutions are shown in Figures 3 and 4 for different gains $h_{1,2}$ of node 1 on channel 2, where $\lambda=1$, $E_{2}=1, h_{1,1}=h_{2,1}=h_{2,2}=1, N_{i}=0.1$ and $D_{i}=D$, $i=1,2$. The results again show that the jamming attack is less successful, if the packet traffic is random and the jammer does not know node 1's queue state information. Node 1 assigns higher traffic rate to a channel with larger gain. As the channel gains approach each other, the rate assignments become equivalent and the total transmission power of node 1 decreases. If the queue state information is not available at jammer, the total power of node 1 (to sustain the target rate and delay) is smaller for all channel gains, and the feasible rate increases, which indicates a performance loss for the jammer.

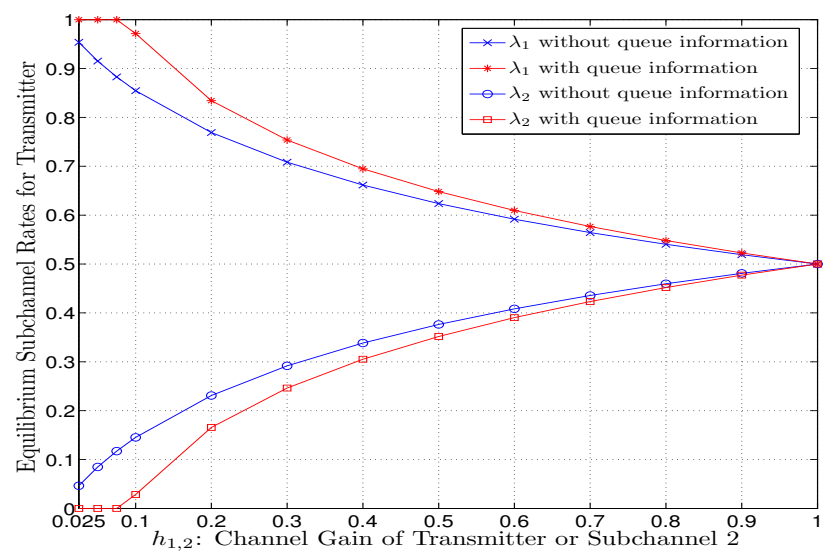

Fig. 3. The equilibrium channel rates of node 1 (where $D=5$ ).

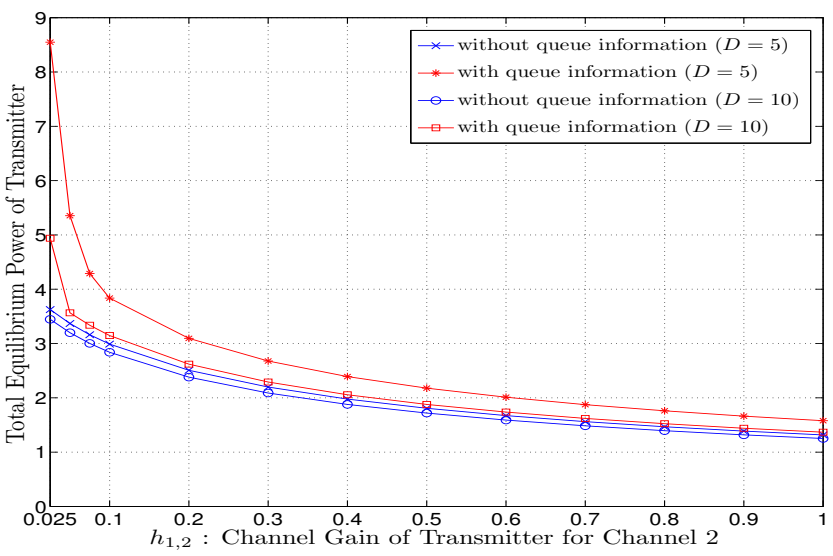

Fig. 4. The total equilibrium transmission power of node 1 .

\section{CONCLUSIONS}

We studied the effects of dynamic traffic on jamming attacks in power controlled MAC. The Nash equilibrium is evaluated for jamming games with delay and energy cost constraints. The results show significant performance loss for jammers (i.e., transmitters improve feasible throughput) if they do not know the queue state information. Dynamic traffic serves as an example that wireless networks are more immune to jamming attacks under uncertainty. This model should be extended to network layer attacks, where the jammer performance depends on the instantaneous availability of packet flows.

\section{REFERENCES}

[1] M. Medard, "Capacity of Correlated Jamming Channels," in Proc. Allerton Conf. Communications, Control and Computing, Oct. 1997.

[2] A. Kashyap, T. Basar, and R. Srikant, "Correlated Jamming on MIMO Gaussian Fading Channels," IEEE Trans. Inform. Theory, vol. 50, no. 9, pp. 2119-2123, Sep. 2004.

[3] S. Shafiee and S. Ulukus, "Capacity of Multiple Access Channels with Correlated Jamming," IEEE Trans. Inform. Theory, vol. 55, no. 10, pp. 4598-4607, Oct. 2009.

[4] E. Altman, K. Avratchenkov, and A. Garnaev, "A Jamming Game in Wireless Networks with Transmission Cost," Lecture Notes in Computer Science, vol. 4465, pp. 1-12, 2007.

[5] — " "Jamming in Wireless Networks under Uncertainty", in Proc. Intl. Symposium on Modeling and Optimization in Mobile, Ad Hoc, and Wireless Networks (WiOpt), Seoul, Korea, June 2009.

[6] R. K. Mallik, R. A. Scholtz, and G. P. Papavassilopoulos, "Analysis of an OnOff Jamming Situation as a Dynamic Game," IEEE Trans. Commun, vol. 48, no. 8, pp. 1360-1373, Aug. 2000.

[7] Y. E. Sagduyu and A. Ephremides, "SINR-based MAC Games for Selfish and Malicious Users," in Proc. Information Theory and Applications Workshop, San Diego, CA, Jan. 2007.

[8] Y. E. Sagduyu, R. Berry, and A. Ephremides, "Wireless Jamming Attacks under Dynamic Traffic Uncertainty," in Proc. Intl. Symposium on Modeling and Optimization in Mobile, Ad Hoc, and Wireless Networks (WiOpt), Avignon, France, 2010.

[9] — "MAC Games for Distributed Wireless Network Security with Incomplete Information of Selfish and Malicious User Types," in Proc. Intl. Conf. on Game Theory for Networks, Istanbul, Turkey, May 2009.

[10] J. Zander, "Jamming in Slotted ALOHA Multihop Packet Radio Networks," IEEE Transactions on Communications, vol. 39, no. 10, pp. 1525-1531, Oct.1991.

[11] R. Berry and E. Yeh, "Cross-layer Wireless Resource Allocation," IEEE Signal Processing Magazine, vol. 21, no. 5, pp. 59-68, Sep. 2004.

[12] D. Bertsekas and R. Gallager, Data Networks. Englewood Cliffs, NJ: Prentice Hall, 1992 